\title{
In-band Network Function Telemetry
}

\author{
Jianzhe Liang, Jun Bi, Yu Zhou, Cheng Zhang* \\ Tsinghua University
}

\section{CCS CONCEPTS}

- Networks $\rightarrow$ Programmable networks;

\section{KEYWORDS}

Network function virtualization, network telemetry

\section{ACM Reference Format:}

Jianzhe Liang, Jun Bi, Yu Zhou, Cheng Zhang. 2018. In-band Network Function Telemetry. In SIGCOMM Posters and Demos '18: ACM SIGCOMM 2018 Conference Posters and Demos, August 2025, 2018, Budapest, Hungary. ACM, New York, NY, USA, 3 pages. https://doi.org/10.1145/3234200.3234236

\section{INTRODUCTION}

Network Function Virtualization (NFV) is now emerging to replace hardware middleboxes with software running on commodity servers, offering tremendous flexibility in the process of life cycle management of network functions. There are currently many excellent platforms for building and running virtual network functions (VNF) such as BESS [3] and NetVM [1], facilitating the life cycle management of VNFs as well as offering a generally good enough performance guarantee [4].

Despite NFV's widespread use in industry, little is known about VNFs' runtime performance, which is of great importance in SLA verification, troubleshooting, and other thirdparty applications. Such monitoring can be more challenging when source code is not available. Even if individual measurements are possible, it is not trivial to design and implement a

\footnotetext{
*Jianzhe Liang, Jun Bi, Yu Zhou and Cheng Zhang are with Network Sciences and Cyberspace, Tsinghua University and Beijing National Research Center for Information Science and Technology (BNRist). This work is supported by National Key R\&D Program of China (2017YFB0801701) and the National Science Foundation of China (No.61472213). Jun Bi is the corresponding author.
}

\footnotetext{
Permission to make digital or hard copies of all or part of this work for personal or classroom use is granted without fee provided that copies are not made or distributed for profit or commercial advantage and that copies bear this notice and the full citation on the first page. Copyrights for components of this work owned by others than ACM must be honored. Abstracting with credit is permitted. To copy otherwise, or republish, to post on servers or to redistribute to lists, requires prior specific permission and/or a fee. Request permissions from permissions@acm.org.

SIGCOMM Posters and Demos '18, August 20-25, 2018, Budapest, Hungary

(C) 2018 Association for Computing Machinery.

ACM ISBN 978-1-4503-5915-3/18/08 ..\$15.00

https://doi.org/10.1145/3234200.3234236
}

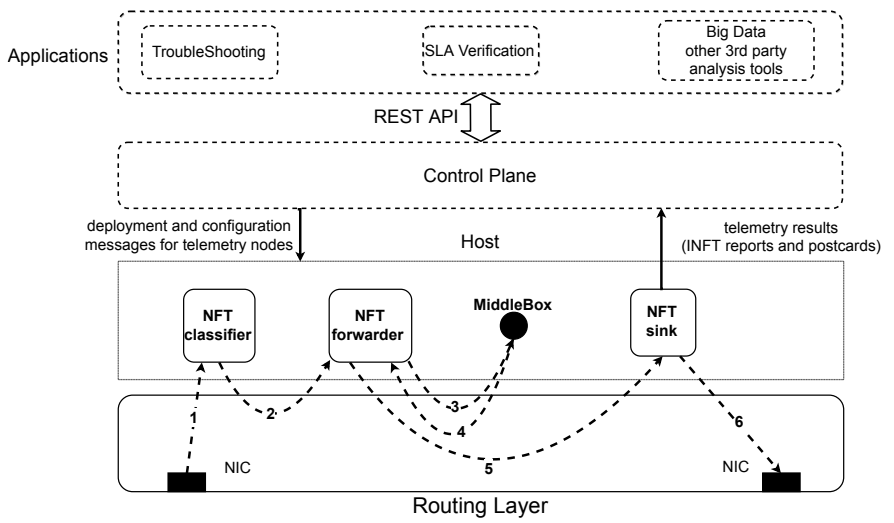

Figure 1: Design overview of NFT.

universal architecture for VNF telemetry with limited overhead. To the best of our knowledge, no work has been done on the design of a comprehensive architecture for efficiently measuring the runtime performance of VNFs. The framework in [5] only focuses on emulation in a development environment. REMAP in [6] is tailored for Service Function Chaining only. The architecture proposed there requires frequent reports of each telemetry node to the control plane, which may cause severe performance degradation.

To effectively monitor runtime performance of NFs, we introduce In-band Network Function Telemetry (INFT). INT (in-band network telemetry) is a framework designed to allow the collection of network state by data plane [2], incurring little overhead (no control plane intervention) and capable of capturing ephemeral changes across network. INFT also relies on data plane for telemetry but aims to become middlebox-transparent.

We present NFT, a lightweight, black-box based, highly configurable telemetry framework. NFT is built directly upon current NFV platforms and requires no modification of measured NFs. The telemetry is still mostly INT in nature, incurring little overhead and capable of monitoring fast-changing performance by exploiting modern CPUs' instruction level parallelism. We add support for postcard-like reports in our framework to allow efficient track of individual packets.

Our contributions are as follows. Firstly, we design a universal architecture capable of measuring middleboxes' performance without modifying them while incurring little overhead. Secondly, we propose the header format for both inband telemetry report and postcard-like report. Finally, elementary evaluation has been performed to prove our design incurs little overhead. 


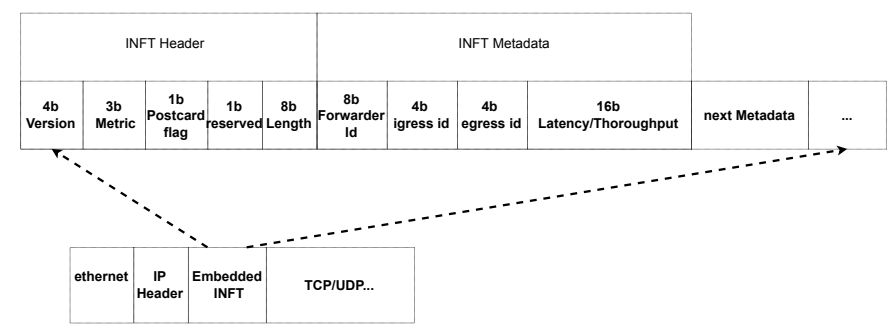

Figure 2: INFT protocol embedded in IP Options field

\section{DESIGN}

As is shown in Figure 1, telemetry nodes in NFT are classified into three categories: classifier, forwarder, and sink. A classifier is a trusted entity that creates and inserts telemetry headers into packets selected according to customer/network specific policy. Forwards collect and report metric information by pushing metadata into data packets or sending postcards according to telemetry headers. They also act as proxies for measured NFs. The sink node extracts the metadata and reshapes the packets into regular data packets.

When a packet is received by a host's Network Interface Card (NIC), it is firstly transmitted to an NFT classifier(arrow 1) via underlying routing mechanisms such as BESS or an Open Vswitch. We refer to such mechanisms as Routing Layer. INFT headers are inserted into packets classified as telemetry packets. These packets are then transmitted to an NFT forwarder (arrow 2). The forwarder writes temporary information (e.g. timestamp) into INFT metadata field according to INFT headers and then sends them to target middleboxes (arrow 3). Packets are transmitted back to forwarder upon exit (arrow 4). The forwarder then computes and writes telemetry results into packets according to previously written data (we assume that INFT headers and metadata are placed so that they will not be touched or removed by middleboxes, and are copied in case of multicasting). Finally, packets with INFT metadata are sent to a sink node (arrow 5). Postcard-like reports generated by forwarders according to INFT headers can also be sent to sink nodes directly. The node then extracts the metadata and reformat the packets into regular data packets. Finally, these regular packets are transmitted out the host through NIC (arrow 6). To boost the performance of the framework, certain nodes, like forwarders, can be implemented directly within data plane.

INFT protocol contains INFT header and INFT metadata just like INT (Figure 2). The Metric field determines the type of metadata to push into each INFT packet. The Length field indicates insertion position for next piece of metadata. A set Postcard bit indicates postcard generation. INFT metadata are 4 bytes entries following the INFT header. Each entry contains the identifier of the forwarder writing the data, the id of port through which the packet enters and leaves the

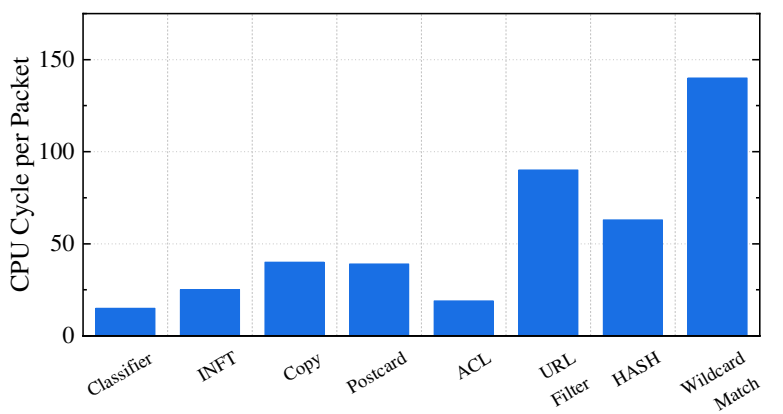

Figure 3: Packet processing rate of different modules.

forwarder and the collected metric data. Currently, we measure latency, I/O packet/byte rate as they are fundamental and straightforward to implement.

The exact position for classifiers to insert INFT protocol and for forwarders to read can be configured by operators, eliminating the influence of adding INFT protocol upon various middleboxes. By default, we suggest embedding the header in the IP Options field since most NFs skip it. Forwarders may also move INFT protocol to other parts of a packet, enabling even more flexible telemetry within data plane. However, as mentioned in evaluation section, such rewrite operation can be costly, leading to performance degradation and thus shall be used with care. Postcard reports contain a Postcard header before the original packet header. The header has a Next Protocol field to indicate the first layer within the packet header. A Sequence field and Left Time field are used to detect packet and postcard drop.

\section{EVALUATION}

We implemented the framework on BESS, i.e. within datap lane and compared its performance with other modules in terms of per-packet processing rate (the difference of Time Stamp Counter upon enterance and exit). All of the modules are run on a single Intel Xeon E5-2620 CPU (2.40GHz).

The results are shown in Figure 3. Classifiers can perform classification pretty fast based on simple rules such as 5tuple (just like the ACL module). Forwarders' significant performance in writing INFT data are also notable. We exploit modern CPU's instruction level parallelism by using conditional moves instead of conditional jumps to avoid CPU bubbles when choosing telemetry data according to INFT header and ingress id.

However, Forwarders take much longer time to move INFT protocols to other parts of packets as it needs to perform more copy actions. Such copy can be avoided in BESS by utilizing reserved buffer. Postcard generation is slow as new packets need to be allocated. Nonetheless, such overhead is acceptable when postcard rate is low. 


\section{REFERENCES}

[1] Jinho Hwang, K. K. Ramakrishnan, and Timothy Wood. 2014. NetVM: High Performance and Flexible Networking Using Virtualization on Commodity Platforms. In Proceedings of the 11th USENIX Conference on Networked Systems Design and Implementation (NSDI'14). USENIX Association, Berkeley, CA, USA, 445-458. http://dl.acm.org/citation. $\mathrm{cfm}$ ?id=2616448.2616490

[2] Changhoon Kim, Parag Bhide, Ed Doe, Hugh Holbrook, Anoop Ghanwani, Dan Daly, Mukesh Hira, and Bruce Davie. 2016. In-band Network Telemetry. (2016). https://p4.org/assets/INT-current-spec.pdf.

[3] Berkeley NetSys Lab. 2015. BESS: Berkeley Extensible Software Switch. Website. (2015). https://github.com/NetSys/bess.

[4] Zhixiong Niu, Hong Xu, Libin Liu, Yongqiang Tian, Peng Wang, and Zhenhua Li. 2017. Unveiling performance of NFV software dataplanes. In Proceedings of the 2nd Workshop on Cloud-Assisted Networking
(CAN'17). ACM, New York, NY, USA, 13-18. https://dl.acm.org/citation. cfm?id $=3158430$

[5] S. v. Rossem, W. Tavernier, M. Peuster, D. Colle, M. Pickavet, and P. Demeester. 2016. Monitoring and debugging using an SDK for NFVpowered telecom applications. In IEEE Conference on Network Function Virtualization and Software Defined Networks (NFV-SDN).

[6] Ming Xia, Meral Shirazipour, Heikki Mahkonen, Ravi Manghirmalani, and Attila Takacs. 2015. Resource Optimization for Service Chain Monitoring in Software-Defined Networks. In Proceedings of the 2015 Fourth European Workshop on Software Defined Networks. 